

\title{
Quantitative Intracranial Atherosclerotic Plaque Characterization at 7T MRI: An Ex Vivo Study with Histologic Validation
}

(D)A.A. Harteveld, (D) N.P. Denswil, (D).C.W. Siero, (D).J.M. Zwanenburg, (D)A. Vink, DB. Pouran, (DW.G.M. Spliet, (DD.W.J. Klomp,

(D) P.R. Luijten, (DM.J. Daemen, (D). Hendrikse, and (D)A.G. van der Kolk

\begin{abstract}
BACKGROUND AND PURPOSE: In recent years, several high-resolution vessel wall MR imaging techniques have emerged for the characterization of intracranial atherosclerotic vessel wall lesions in vivo. However, a thorough validation of MR imaging results of intracranial plaques with histopathology is still lacking. The aim of this study was to characterize atherosclerotic plaque components in a quantitative manner by obtaining the MR signal characteristics ( $T 1, T 2, T 2^{*}$, and proton density) at $7 T$ in ex vivo circle of Willis specimens and using histopathology for validation.
\end{abstract}

MATERIALS AND METHODS: A multiparametric ultra-high-resolution quantitative MR imaging protocol was performed at 7T to identify the MR signal characteristics of different intracranial atherosclerotic plaque components, and using histopathology for validation. In total, 38 advanced plaques were matched between MR imaging and histology, and ROI analysis was performed on the identified tissue components.

RESULTS: Mean $T 1, T 2$, and $T 2 *$ relaxation times and proton density values were significantly different between different tissue components. The quantitative 77 map showed the most differences among individual tissue components of intracranial plaques with significant differences in $T 7$ values between lipid accumulation ( $T 7=838 \pm 167 \mathrm{~ms})$, fibrous tissue $(T 7=583 \pm 161 \mathrm{~ms})$, fibrous cap $(T 7=481 \pm 98 \mathrm{~ms})$, calcifications $(T 7=314 \pm 39 \mathrm{~ms})$, and the intracranial arterial vessel wall $(T 7=436 \pm 122 \mathrm{~ms})$.

CONCLUSIONS: Different tissue components of advanced intracranial plaques have distinguishable imaging characteristics with ultrahigh-resolution quantitative MR imaging at 7T. Based on this study, the most promising method for distinguishing intracranial plaque components is Tl-weighted imaging.

ABBREVIATIONS: COW = circle of Willis; DESPOT1 = driven equilibrium single pulse observation of $T 7$; DESPOT2 = driven equilibrium single pulse observation of $T 2 ; \mathrm{PD}=$ proton density

schemic stroke is one of the major diseases in the Western world, associated with high morbidity and mortality. ${ }^{1,2}$ Identifying the cause of ischemic stroke is of great clinical importance, not only for deciding the best treatment options for the individual

Received August 19, 2015; accepted after revision October 27.

From the Departments of Radiology (A.A.H., J.C.W.S., J.J.M.Z., D.W.J.K., P.R.L., J.H., A.G.v.d.K.), Pathology (A.V., W.G.M.S.), Orthopedics (B.P.), and Rheumatology (B.P.), and Image Sciences Institute (I.J.M.Z.), University Medical Center Utrecht, Utrecht, the Netherlands; Department of Pathology (N.P.D., M.J.D.), Academic Medical Center, Amsterdam, the Netherlands; and Department of Biomedical Engineering (B.P.), Faculty of Mechanical, Maritime, and Materials Engineering, Delft University of Technology, Delft, the Netherlands.

This work was supported by the Netherlands Organization for Scientific Research under grant 91712322 (J.H.).

Please address correspondence to A.A. Harteveld, MSc, Department of Radiology, University Medical Center Utrecht, Box 85500, 3508 GA Utrecht, the Netherlands, e-mail a.a.harteveld-2@umcutrecht.nl.

-- Indicates open access to non-subscribers at www.ajnr.org

三 Indicates article with supplemental on-line table.

http://dx.doi.org/10.3174/ajnr.A4628 patient but also for possibly preventing future ischemic events, both recurrent and new.

Intracranial atherosclerosis is one of the main causes of ischemic stroke and TIA worldwide, accounting for approximately $9 \%-33 \%$ of all ischemic strokes and TIAs (depending on raceethnicity) and is the major cause of cerebral ischemic events in the Asian population. Additionally, the risk of recurrent ischemic stroke is increased in patients with underlying intracranial atherosclerosis. $^{3-8}$ Similar to extracranial atherosclerosis, for intracranial atherosclerosis, the degree of luminal stenosis is currently the main factor determining whether an atherosclerotic plaque is symptomatic and/or treatment is necessary. In the past decade, plaque composition has become an additional important feature in extracranial atherosclerosis management, enabling identification of specific culprit lesions requiring treatment, even when not causing significant stenosis. ${ }^{9-12}$ However, for intracranial atherosclerosis, this is not yet common practice, even though the literature suggests that also intracranially, stenosis grade is not always 
Table 1: Scan parameters of the 7T MRI protocol

\begin{tabular}{lccc}
\hline \multicolumn{1}{c}{ Pulse Sequence } & DESPOT1 & DESPOT2 & T2* Map \\
\hline TR/TE1/TE2 (ms) & $16 / 4.3 /-$ & $36 / 18 /-$ & $53 / 6 / 26$ \\
Flip angle & $11^{\circ} / 44^{\circ}$ & $12^{\circ} / 62^{\circ}$ & $29^{\circ}$ \\
No. of sections & 153 & 153 & 153 \\
FOV (mm) & $150 \times 150 \times 20$ & $150 \times 150 \times 20$ & $150 \times 150 \times 20$ \\
Acquisition matrix & $1152 \times 1154$ & $1152 \times 1152$ & $1152 \times 1154$ \\
Acquired voxel & $0.13 \times 0.13 \times 0.13$ & $0.13 \times 0.13 \times 0.13$ & $0.13 \times 0.13 \times 0.13$ \\
$\quad$ size (mm) & & & \\
Bandwidth (Hz/pixel) & 165 & 41 & 101 \\
TFE factor & 1154 & 1152 & 1154 \\
Repeated scans & 4 & 3 & 1 \\
Acquisition time & $06 \mathrm{hr} 21 \mathrm{~min} 28 \mathrm{sec}$ & $11 \mathrm{hr} 07 \mathrm{~min} 34 \mathrm{sec}$ & $02 \mathrm{hr} \mathrm{00} \mathrm{min} \mathrm{53} \mathrm{sec}$ \\
\hline Note:-TFE indicates turbo field echo & & &
\end{tabular}

associated with a risk of ischemic events: In patients with a highgrade stenosis, only 1 in every $5-10$ patients will have a recurrent ischemic stroke. ${ }^{5,6,13,14}$ This implies that like in extracranial atherosclerosis, luminal information is not the only important marker for future cerebrovascular events.

In recent years, several high-resolution intracranial vessel wall imaging techniques using 3T and 7T MR imaging have emerged for the evaluation and characterization of atherosclerotic vessel wall lesions in vivo. ${ }^{15}$ However, these in vivo techniques so far have not been validated with histopathology, due to poor accessibility of the intracranial arteries. Therefore, the question arises if these techniques are truly able-ie, have enough image contrast-to distinguish different atherosclerotic plaque components. In the past 2 years, 2 ex vivo correlation studies have been performed at 7T MR imaging to assess its ability to visualize different intracranial atherosclerotic plaque components. ${ }^{16,17}$ These ex vivo studies showed that 7T MR imaging is capable of identifying focal thickening of the intracranial arterial vessel wall and distinguishing different plaque components within advanced intracranial atherosclerotic plaques with different image contrast weightings. Recently, a first case report was published demonstrating the correlation between certain intracranial atherosclerotic plaque components visualized in vivo at $3 \mathrm{~T}$ and histologic validation of the plaque postmortem. ${ }^{18}$ Those studies used qualitative MR images to score the atherosclerotic plaque signal heterogeneities. As a next step toward validation, quantitative assessment of MR signal characteristics of specific plaque components might enable more firm conclusions regarding the ability of T1-, $\mathrm{T} 2-$, $\mathrm{T}^{*}$-, and proton density (PD)-weighted sequences in characterizing intracranial atherosclerotic plaques. Once it is known which plaque components can be identified with ex vivo MR imaging sequences, a translation can be made to in vivo intracranial vessel wall MR imaging, by developing sequences based on the nuclear magnetic resonance tissue properties of the identified atherosclerotic plaque components. In the current study, a multiparametric ultra-high-resolution quantitative MR imaging protocol was performed to identify the MR signal characteristics of different intracranial atherosclerotic plaque components, and using histopathology for validation.

\section{MATERIALS AND METHODS \\ Specimens}

Human circle of Willis (CoW) specimens with a macroscopic presence of high atherosclerotic plaque burden were selected by an experienced pathologist (A.V.) from $>100$ postmortem examinations that were performed in our institution. Material was handled in a coded manner that met the criteria of the Code of Conduct used in the Netherlands for the responsible use of human tissue in medical research (www.federa.org/codes-conduct), and institutional review board approval for this retrospective study was obtained.

\section{Specimen Preparation}

All specimens had been stored in buffered formalin (4\%). Preparation of the specimens for MR imaging and histologic sampling was performed according to the method previously described by van der Kolk et al. ${ }^{16}$ Cactus spines were used as fiducials and placed at 15 locations in the agarose gel adjacent to an artery for histologic sampling, to enable spatial correlation with histology. The 15 locations were chosen to sample all major vessels of the CoW, including their branches (eg, A2 segment of the anterior cerebral artery), and the fiducials were placed at the location of a visible vessel wall abnormality (if present). The locations included the following: the anterior cerebral (A1 and A2 segments), middle cerebral (M1 segment), intracranial internal carotid (C7 segment), posterior cerebral (P2 segment), basilar (upper, middle, and lower part), and vertebral arteries (left and right if applicable).

\section{Imaging}

The embedded specimens were scanned in a 7T whole-body system (Achieva; Philips Healthcare, Best, the Netherlands). A custom-made high density receive coil ( 16 channels per $70 \mathrm{~cm}^{2}$; High density surface array; MR Coils BV, Drunen, the Netherlands) was used for signal reception, ${ }^{19}$ and a volume transmit/receive coil, for transmission (Quad TR; Nova Medical, Wilmington, Massachusetts). Each embedded specimen was imaged individually. The petri dish containing the embedded specimen was placed in the middle of the transmit coil on top of the receive coil; plastic filling was used to stabilize and secure the petri dish.

\section{Protocol}

Imaging was performed at room temperature. The scan protocol had 3 sequences with different contrast weightings to image the specimens, from which quantitative MR parameter maps were calculated. For $T 1, T 2$, and $P D$ mapping, the driven equilibrium single pulse observation of T1 (DESPOT1) and T2 (DESPOT2) sequences were used. ${ }^{20} \mathrm{TR}$ was chosen as short as allowed by the desired spatial resolution and FOV. We calculated optimal flip angle pairs for the DESPOT1 and DESPOT2 scans, assuming a range of $T 1$ and $T 2$ values of $150-650 \mathrm{~ms}$ and $10-30 \mathrm{~ms}$, respectively. $T 2^{*}$ maps were obtained by using a dual-echo $3 \mathrm{D} \mathrm{T} 2{ }^{*}$ weighted scan. The applied scan parameters are presented in Table 1 . A $3 \mathrm{D}_{1}$ map was acquired by using the actual flip angle method to correct for $B_{1}$ inhomogeneity during postprocessing of the MR images. ${ }^{21}$ Total scan time was approximately 19.5 hours per specimen. All MR images were acquired with an isotropic resolution. This allowed reconstructions along arbitrary planes, 
which enabled accurate matching of the MR images to the sectioning plane of the histologic sections.

\section{MR Image Processing}

The MR images were processed by using Matlab, Version R2014b (MathWorks, Natick, Massachusetts). First, the DESPOT1 and DESPOT2 dynamic scans were coregistered to correct for small shifts between the matching images. Next, the parametric images $\left(T 1, T 2, T 2^{*}\right.$ relaxation times and $P D$ values) were calculated from the original MR imaging datasets according to the method previously described by Deoni et al. ${ }^{20} \mathrm{~B}_{1}$ correction was performed during calculation of the parametric $T 1$ and $T 2$ maps, to correct for $B_{1}$ inhomogeneity within the specimen.

\section{Histopathology}

After MR imaging, 0.5-mm-thick tissue samples were taken from the 15 marked locations of each CoW specimen for histologic processing, which was performed by using an in-housedeveloped protocol, as previously described. ${ }^{16}$ Classification of the histologic sections was performed according to the modified American Heart Association classification by Virmani et $\mathrm{al},{ }^{22}$ a well-established method that is based on atherosclerotic characteristics as follows: 1) no anomaly; 2) early lesion, including intimal thickening ( $<50 \%$ smooth-muscle cells, no lipids, inflammatory cells), fatty streak, and pathologic intimal thickening ( $>50 \%$ smooth-muscle cells, rich in proteoglycans, foamy macrophages); and 3) advanced lesion, including fibrolipid plaque ( $>40 \%$ lipid), thin cap atheroma $(<65-\mu \mathrm{m}$ thickness), fibrous plaque $(<40 \%$ lipid), fibrocalcified plaque ( $>40 \%$ calcified), and calcified nodule (calcified element protruding the intima). When applicable, plaque complications (rupture, hemorrhage, or erosion) were also assessed. The histologic classification was performed by 1 experienced observer (N.P.D.), blinded to the MR imaging results.

\section{Matching of MR and Histology Images}

Image reconstructions were made perpendicular to the relevant arteries (section thickness, $0.13 \mathrm{~mm}$ ) at the locations of the fiducials seen on the MR images, by using the software program VesselMASS (Leiden University Medical Center, Leiden, the Netherlands). The MR images and the histologic sections were manually matched by 1 observer (A.A.H.) by using the marked locations with the fiducials in the MR images, the ink markings in the histologic sections, and gross morphologic features. Samples were excluded when no match was found or when air bubbles were present in the MR images.

\section{Plaque Component Analysis}

To analyze different plaque components, we used only plaques histologically scored as advanced, therefore including several components, because a previous study showed no heterogeneity in qualitative signal intensity in early lesions. ${ }^{16}$ The identified plaque components included fibrous tissue, fibrous cap, lipid accumulation (increasing mass of lipids [lipid retention] within the intimal area, which is defined as the "plaque"), intraplaque hemorrhage, and calcifications (deposition of extracellular calcium within the plaque). On the basis of the identified plaque compo- nents in a histologic section, ROIs were drawn manually within the corresponding regions on T1-weighted MR images (DESPOT1 acquired with a flip angle of $44^{\circ}$, used for calculation of the quantitative maps) by 1 observer (A.A.H.). The ROIs were used to calculate the mean $T 1, T 2$, and $T 2^{*}$ relaxation times and $P D$ values for those regions in the MR parameter maps, by using the dedicated vessel wall analysis software VesselMASS.

\section{Statistical Analysis}

All statistical analyses were performed by using SPSS statistics (Version 20.0; IBM, Armonk, New York). Because the data were normally distributed but the assumption of homogeneity of variance was not met, the Welch test was conducted to compare the mean ROI values of the different tissue components. GamesHowell post hoc tests were performed for pair-wise comparisons. A $P$ value $<.05$ was statistically significant.

\section{RESULTS}

Fifteen CoW specimens were selected for this study ( 9 men; mean age, $68.7 \pm 13.3$ years). In total, 213 samples of CoW arteries were obtained (in 8 specimens, $\geq 1$ vessel segment was absent; $n=12$ vessel segments); 8 of the 213 samples (3.8\%) were excluded because they were not assessable for histologic classification (the samples could not be sliced properly), resulting in 205 samples available for histologic classification.

\section{Histologic Classification}

Six samples (2.9\%) were scored as nondiseased; 143 samples (69.8\%) showed early lesions (intimal thickening, $n=97$, pathologic intimal thickening, $n=19$, and fatty streak, $n=27$ ); and 56 samples $(27.3 \%)$ showed advanced plaques (fibrous plaque, $n=$ 28 , fibrolipid plaque, $n=25$, and fibrocalcified plaque, $n=3$ ). No calcified nodules or thin cap atheromas were seen. Of the 56 advanced plaques, $18(32 \%)$ were excluded due to lack of a match $(n=3)$ or an air bubble present in the MR images $(n=15)$, resulting in $38 \mathrm{MR}$ imaging-matched advanced atherosclerotic plaques (20 fibrous plaques, 16 fibrolipid plaques, and 2 fibrocalcified plaques) that could be used for the ROI analysis. ROIs were drawn in the matched MR images of the advanced atherosclerotic plaques within the corresponding regions of the tissue components identified in the histologic sections: lipid accumulation $(n=17)$, fibrous tissue $(n=24)$, fibrous cap $(n=12)$, calcifications $(n=5)$, and vessel wall $(n=37)$.

\section{Quantitative MR Parameter Maps}

Figures 1-3 show examples of histologic sections with the matched MR parameter maps $\left(T 1, T 2, T 2^{*}\right.$, and $\left.P D\right)$ of advanced plaques from the CoW specimens. Figure 4 and Table 2 show the results of the ROI analysis. For each quantitative MR parameter map, a significant difference was found among certain identified tissue components. In Fig 3B, the single sample containing intraplaque hemorrhage is shown. The hemorrhage can be distinguished from the surrounding fibrous tissue in the T1 and $P D$ maps. This sample was not included in the statistical analysis because only 1 sample contained an intraplaque hemorrhage in this study. 


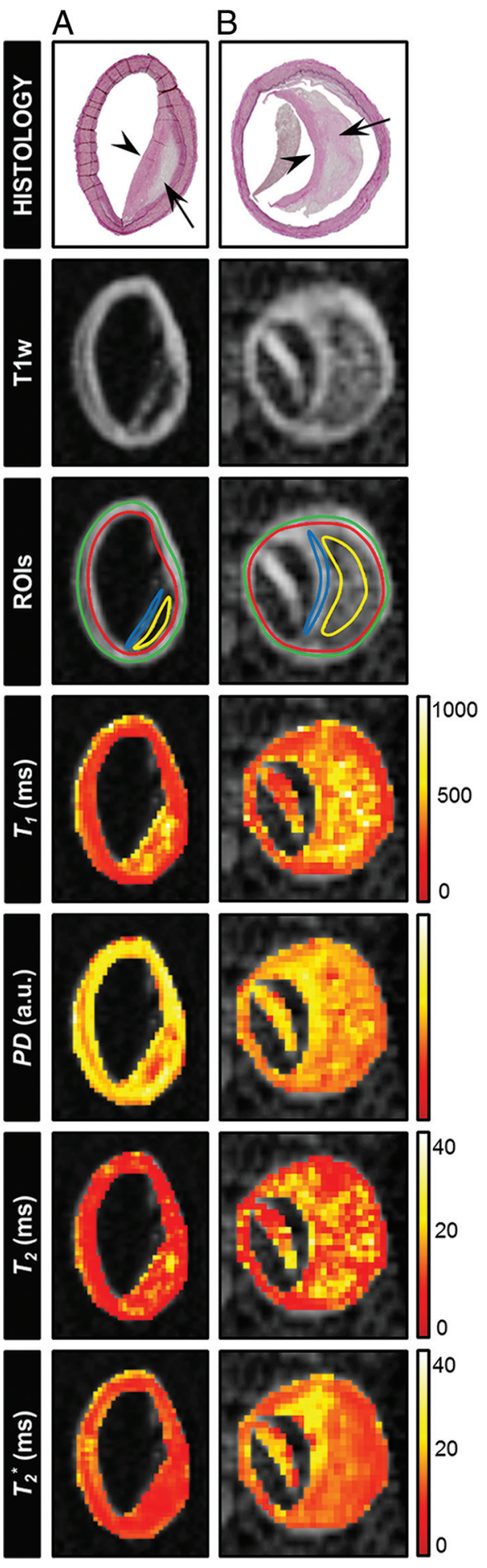

FIG 1. Histologic sections (Van Gieson elastic stain) of 2 different samples from the CoW specimens classified as fibrolipid plaques: $(A)$ left vertebral artery and $(B)$ left middle cerebral artery (arrow indicates lipid accumulation; arrowhead, fibrous cap). The corresponding T1-weighted image (T7w; DESPOT1 acquired with a flip angle of $44^{\circ}$ ) and the calculated parametric maps $\left(T 1, T 2, T 2^{*}\right.$, and $\left.P D\right)$ are shown. ROIs were drawn at the location of tissue components identified in the histologic section (yellow ROI indicates lipid accumulation; blue $\mathrm{ROI}$, fibrous cap; green + red ROI, vessel wall) to calculate the mean $T 1, T 2, T 2^{*}$, and $P D$ values for those regions. Lipid accumulation can be distinguished from the fibrous cap and the vessel wall in the $T 7, P D$, and $T 2$ maps. A, The lipid accumulation area has already developed into the beginning of a lipid core.

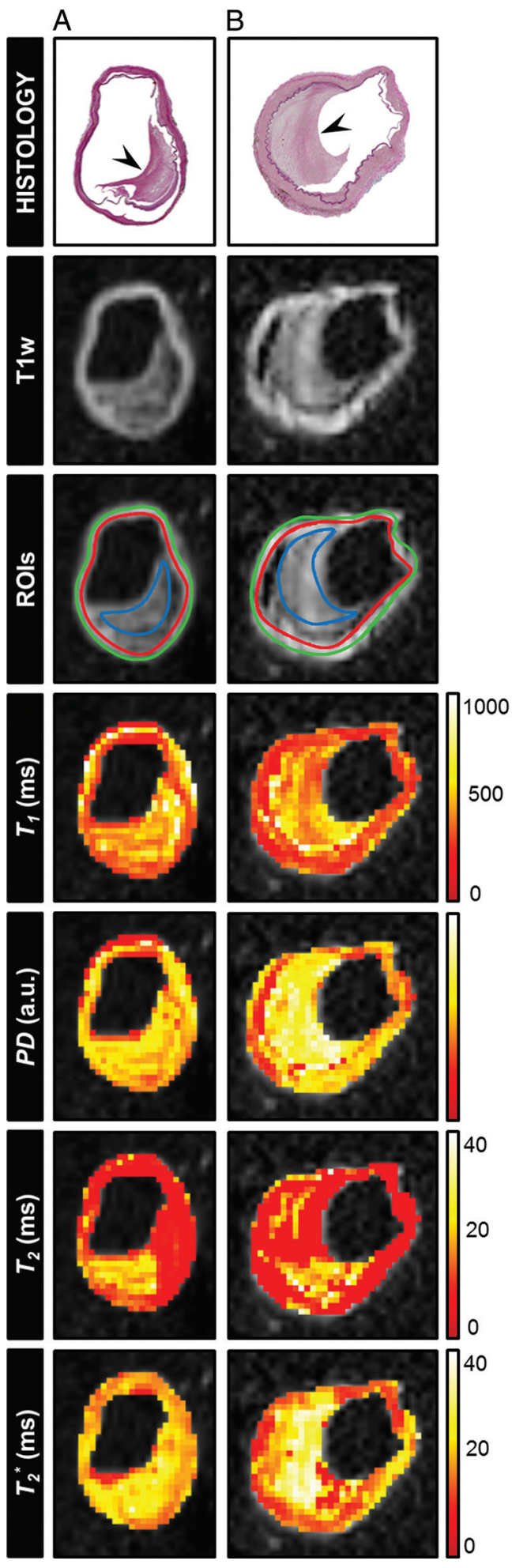

FIG 2. Histologic sections (Van Gieson elastic stain) of 2 different samples from the CoW specimens classified as fibrous plaques: $(A)$ right middle cerebral artery and $(B)$ left intracranial internal carotid artery (arrowhead indicates fibrous tissue). The corresponding T7weighted image (DESPOT1 acquired with a flip angle of $44^{\circ}$ ) and the calculated parametric maps $\left(T 1, T 2, T 2^{*}\right.$, and $\left.P D\right)$ are shown. ROIs were drawn at the location of tissue components identified in the histologic section (blue ROI indicates fibrous tissue; green + red ROI, vessel wall) to calculate the mean $T 1, T 2, T 2^{*}$, and $P D$ values for these regions. Here, fibrous tissue can be distinguished from the vessel wall in the $T 7$ and $T 2$ maps (partly) and, to a lesser extent, in the $T 2^{*}$ map. 


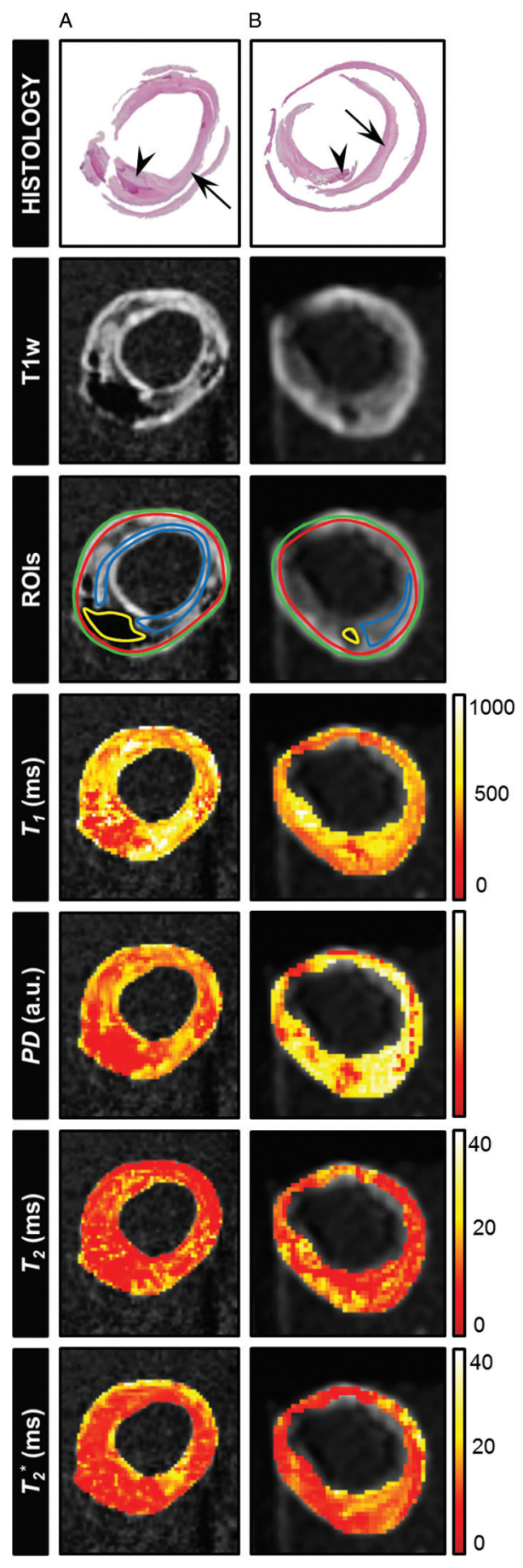

FIG 3. Histologic sections (H\&E stain) of 2 different samples from the CoW specimens containing calcifications $(A)$ and an intraplaque hemorrhage (B): right intracranial internal carotid artery $(A)$, classified as fibrocalcified plaque, and middle part of the basilar artery $(B)$, classified as fibrous plaque with an old hemorrhage (arrow indicates fibrous tissue, arrowhead: calcification, $A$, and hemorrhage, $B$ ). The corresponding Tl-weighted image (DESPOTl acquired with a flip angle of $\left.44^{\circ}\right)$ and the calculated parametric maps $\left(T 1, T 2, T 2^{*}\right.$, and $\left.P D\right)$ are shown. ROIs were drawn at the location of tissue components identified in the histologic section (yellow ROI indicates calcification, $A$, or intraplaque hemorrhage, $B$; blue ROI, fibrous tissue; green + red ROI, vessel wall) to calculate the mean $T 7, T 2, T 2^{*}$, and $P D$ values for those regions. Here, the tissue area containing calcifications can be well distinguished in both the $P D$ and 77 maps and, to a lesser extent, in the $T 2$ and $T 2^{*}$ maps. The intraplaque hemorrhage can be distinguished from the surrounding fibrous tissue in the $T 7$ and $P D$ maps.

\section{Post Hoc Analysis}

The On-line Table gives an overview of the post hoc tests performed for pair-wise comparisons of mean ROI values of the identified tissue components, with the resulting $P$ values.

\section{DISCUSSION}

The current study focused on obtaining ex vivo quantitative $T 1$, $T 2$, and $T 2^{*}$ relaxation times and $P D$ values for different intracranial atherosclerotic plaque components. Its goal was to provide detailed insight into the ability of MR imaging to characterize intracranial atherosclerotic plaque components. Our main results show that primarily, $T 1$ relaxation times were significantly different between different plaque components; the other quantitative maps $\left(P D, T 2\right.$, and $\left.T 2^{*}\right)$ showed mostly nonsignificant differences between the individual tissue components present in the advanced intracranial plaques.

Thus far, atherosclerotic plaques have been extensively studied in extracranial carotid arteries with both in vivo ${ }^{23-25}$ and ex vivo $^{26-29} \mathrm{MR}$ imaging. These studies showed good correlation between MR imaging plaque characteristics and histologic findings. The advantage of extracranial carotid artery plaques is that in vivo MR imaging can be performed before a carotid endarterectomy procedure, allowing direct correlation with histopathology. However, extrapolation of MR signal characteristics of extracranial plaques to intracranial plaques may not be directly applicable because the intracranial arteries are smaller and have some unique histologic features. ${ }^{18,30-32}$ Even so, several studies have recently reported visualization of different intracranial plaque components in vivo by using MR imaging, such as lipids, ${ }^{33}$ fibrous cap, ${ }^{33-35}$ and intraplaque hemorrhage. ${ }^{34,36-38}$ These in vivo techniques, however, have not been validated thoroughly with histopathology, due to the poor accessibility of the intracranial arteries. Recently, Turan et al ${ }^{18}$ published a first case report demonstrating the correlation between certain intracranial atherosclerotic plaque components visualized in vivo at $3 \mathrm{~T}$ with histologic validation of the plaque postmortem. However, further correlations are needed to define additional plaque components and to increase the number of cases. As a first step toward MR imaging validation, 2 ex vivo correlation studies ${ }^{16,17}$ assessed the ability of 7T MR imaging to image plaque components. These qualitative studies showed 7T to have sufficient image contrast to distinguish different plaque components within advanced plaques. As a second step toward validation, the current study quantitatively assessed intracranial atherosclerotic plaques to enable more firm conclusions regarding the ability of T1-, T2-, T2*-, and PDweighted sequences to characterize intracranial atherosclerotic plaques.

Our results showed that the $T 1$ relaxation times gave the most differences between individual tissue components of advanced intracranial plaques. Tissue components identified in the histologic sections as lipid accumulation, fibrous tissue, fibrous cap, calcifications, and vessel wall showed significant differences in $T 1$ relaxation times, indicating that they can be distinguished from each other in a T1-weighted sequence. Lipid accumulation showed the longest mean $T 1$ relaxation time, indicating that this may appear as a hypo-isointense region in a T1-weighted sequence. van der Kolk et al ${ }^{16}$ also 

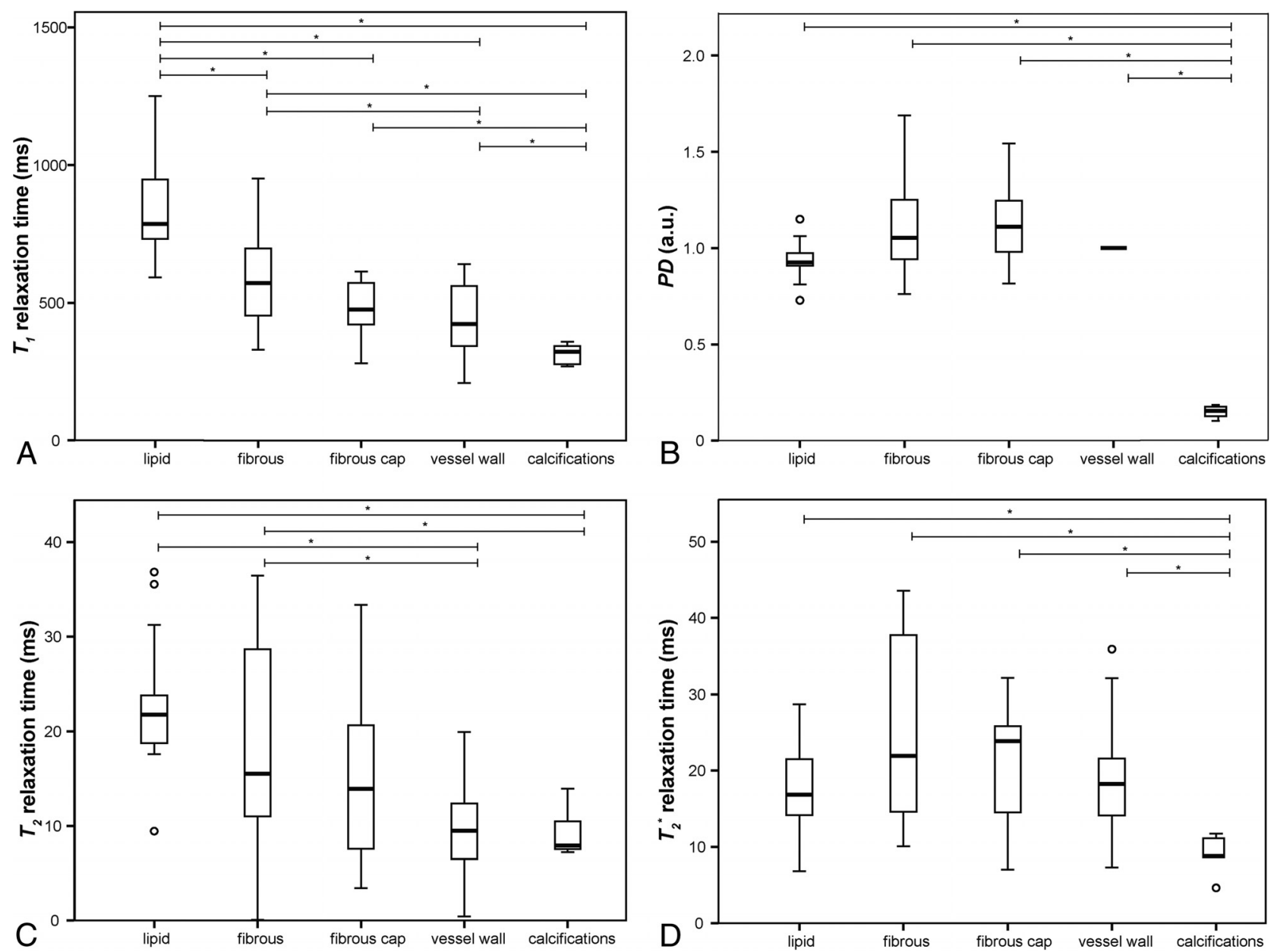

FIG 4. Boxplots showing the distribution of the mean ROI values of each identified tissue component determined in the quantitative $T 7(A), P D$ $(B), T 2(C)$, and $T 2^{*}(D)$ maps of each advanced plaque sample. The asterisk indicates post hoc pair-wise comparison $(P<.05)$.

Table 2: NMR characteristics of different intracranial plaque tissue components ${ }^{a}$

\begin{tabular}{lccccc}
\hline & $\begin{array}{c}\text { Lipid Accumulation } \\
(\boldsymbol{n}=\mathbf{1 7})\end{array}$ & $\begin{array}{c}\text { Fibrous Tissue } \\
(\boldsymbol{n}=\mathbf{2 4})\end{array}$ & $\begin{array}{c}\text { Fibrous Cap } \\
(\boldsymbol{n}=\mathbf{1 2})\end{array}$ & $\begin{array}{c}\text { Calcifications } \\
(\boldsymbol{n}=\mathbf{5})\end{array}$ & $\begin{array}{c}\text { Vessel Wall } \\
(\boldsymbol{n}=\mathbf{3 7})\end{array}$ \\
\hline$T 7(\mathrm{~ms})$ & $838 \pm 167$ & $583 \pm 161$ & $481 \pm 98$ & $314 \pm 39$ & $436 \pm 122$ \\
$P D^{\mathrm{b}}$ & $0.94 \pm 0.10$ & $1.10 \pm 0.26$ & $1.13 \pm 0.21$ & $0.15 \pm 0.03$ & $1.0 \pm 0$ \\
$T 2(\mathrm{~ms})$ & $22 \pm 6.8$ & $18 \pm 10$ & $15 \pm 9.2$ & $9.4 \pm 2.8$ & $10 \pm 4.4$ \\
$T 2^{*}(\mathrm{~ms})$ & $18 \pm 6.2$ & $25 \pm 11$ & $21 \pm 8.4$ & $8.9 \pm 3.0$ & $18 \pm 6.5$ \\
\hline
\end{tabular}

Note:-NMR indicates nuclear magnetic resonance.

a For each identified plaque component the mean \pm SD of the mean ROI values for all parameters $\left(T 7, P D, T 2, T 2^{*}\right)$ are given. Group differences between tissue components were tested with the Welch test; all maps showed a significant difference $(P<.001)$ between tissue components.

${ }^{\mathrm{b}}$ Normalized to vessel wall.

showed the lipid-rich core to be hypointense on T1-weighted imaging of ex vivo CoW specimens at $7 \mathrm{~T}$, while Turan et al ${ }^{18}$ demonstrated in vivo lipid and loose matrix to have an isointense signal intensity compared with the surrounding tissue on a T1-weighted image at 3T. However, both studies were based on a limited number of observations. Previous studies on carotid atherosclerotic plaques ${ }^{11,27,39}$ have shown the lipid-rich core to be hyperintense on T1-weighted imaging. An explanation for this difference could be that the stage of development of the lipid-rich core in the current study might be earlier compared with the extracranial carotid artery studies. In the properties of the lipids. ${ }^{27}$

current study, only 1 sample was identified showing the beginning of a lipid core (Fig 1A); all other samples that were classified as a fibrolipid plaque were still at the stage of lipid accumulation. Intracranial arteries showed more stable lesions and develop mainly as fibrous plaques. ${ }^{30}$ Another explanation could be that lipids in an atherosclerotic plaque undergo a partially irreversible phase transition when cooled $^{40}$; however, it is not clear whether this transition affects the MR imaging

The other quantitative maps $\left(P D, T 2\right.$, and $\left.T 2^{*}\right)$ showed less significant differences among the mean ROI values of the individual tissue components present in the advanced intracranial plaques. The fibrous cap and fibrous tissue showed a larger mean $P D$ value compared with lipid accumulation; however, these differences did not reach statistical significance. Chung et al ${ }^{34}$ also found the fibrous cap to be hyperintense in PD-weighted imaging of intracranial plaques in vivo. Based on the T2 relaxation time, a distinction can be made between lipids/fibrous tissue on the one hand and vessel wall/calcifications on the other. However, for 
each analyzed tissue component, the $T 2$ relaxation times showed a large relative SD. In addition, the $T 2$ relaxation times were not consistently larger than the $T 2^{*}$ relaxation times; this finding is in contrast to what is known from nuclear magnetic resonance theory. The DESPOT2 method for estimating the T2 relaxation times might be more sensitive to noise than the DESPOT1 method and might, thus, have been negatively affected by the limited SNR resulting from the high resolution used in the current study. Future studies specifically focused on plaque $T 2$ relaxation times may show more significant results. For carotid plaque characterization, it has been shown that reviewing multiple contrastweighted images provided the most comprehensive evaluation for certain plaque components (eg, intraplaque hemorrhage and lipid-rich necrotic core). ${ }^{27,41}$

Only at a few locations were intracranial calcified plaques detected. This finding is in agreement with the low prevalence of intracranial calcified plaques beyond the proximal segments of the ICA and vertebral artery. ${ }^{30,42}$ The calcifications that were detected showed low values in all quantitative maps. The PD map showed the largest differences between calcifications and other plaque components and the intracranial vessel wall. It would be expected that this large difference would also be present on the $T 2^{\star}$ maps, due to the increased susceptibility effects; however, this difference was not seen in our small sample. This outcome could be due to the relatively lower SNR for the T2* maps (only 1 signal average was used for the $\mathrm{T} 2^{\star}$-weighted sequence). Also, the $T 2^{\star}$ of calcifications is known to be very short relative to the shortest TE of the current $\mathrm{T} 2^{\star}$-weighted sequence. ${ }^{43}$ These factors can potentially impede accurate fitting of the $T 2^{*}$ relaxation times. A sequence can be used that measures at even shorter TEs to overcome this impediment; however, this result would come at a price of attainable spatial resolution.

Plaque complications were very rare in this study: Only 1 sample with an intraplaque hemorrhage was found. Previous studies have shown a lower prevalence of intraplaque hemorrhage in intracranial compared with extracranial atherosclerosis, and the prevalence of intraplaque hemorrhage is higher in plaques of symptomatic patients (who experienced a cerebral infarct) compared with asymptomatic patients. ${ }^{30,31,38}$ In the current study, the CoW specimens were selected on the basis of the macroscopic presence of a high atherosclerotic plaque burden, without knowledge of the clinical background of the patients. In addition, samples were taken at 15 marked locations per CoW specimen only. Increasing the number of samples per specimen and selecting specimens from symptomatic patients might increase the number of plaque complications found.

This study has several limitations. First, fixation effects of formalin have been shown to change relaxation parameters with time $^{44,45}$ Compared with relaxation times in vivo for the vessel wall of the extracranial carotid arteries, ${ }^{46}$ the relaxation times in the current ex vivo study are considerably shorter. Similar shortening was found in a previous postmortem study, in which the relaxation times for gray and white matter were measured in formalin-fixed human brains. ${ }^{47}$ With an understanding of the effects of sample preparation, the MR imaging data can be correctly related to in vivo data. ${ }^{44}$ Second, the MR imaging experiments were performed at room temperature instead of body temperature, which will have influenced the quantitative measurements in the postmortem specimens. ${ }^{44}$ The 7T MR imaging platform does not provide the possibility of performing temperature-controlled imaging. For now, this is the first study trying to identify specific intracranial plaque components in a quantitative manner to measure whether individual tissue components can be distinguished from each other on the basis of their nuclear magnetic resonance tissue properties. This might suggest which contrast weightings need further consideration, to offer the greatest potential to really discriminate different plaque components in vivo. A next step for future studies would be to validate the current findings in a new set of intracranial plaques with optimized MR imaging sequences, preferably of patients with cerebrovascular disease, by characterizing the plaque components on the basis of the MR images alone (with histologic validation afterward) and assessing the reliability of the measurements.

This study was performed to investigate which MR imaging relaxation times discriminate best among the different plaque components and, thereby, in essence, to predict which image contrast weighting would work best in vivo in characterizing atherosclerotic plaques. On the basis of this study, the $T 1$ relaxation time seems to be the most promising parameter. For now, a direct translation of the quantitative sequences used in the current ex vivo setting to in vivo will be difficult due to the long scan times necessary to achieve the ultra-high resolution required to identify different plaque components. However, this study shows that T1weighted imaging is probably the best method for distinguishing intracranial plaque components ex vivo and therefore might be most promising for in vivo application. Now that it is known which plaque components can be identified with ex vivo MR imaging sequences, a translation can be made to in vivo intracranial vessel wall MR imaging by fine-tuning the current in vivo sequences on the basis of the nuclear magnetic resonance tissue properties of the identified atherosclerotic plaque components to obtain optimal image contrast between the different tissue components. Also, future developments in receive coil technology (increasing the number of receive elements) may provide significant improvement in SNR to make quantitative assessment of the intracranial arterial vessel wall possible in vivo. Furthermore, in vivo, we expect that quantitative assessment will first become feasible for the thicker arterial vessel wall of the more proximal intracranial arteries, including the distal carotid artery and the vertebrobasilar arterial vessel walls.

\section{CONCLUSIONS}

We showed that different tissue components of advanced intracranial plaques have distinguishable imaging characteristics by using ultra-high-resolution quantitative MR imaging at 7T. Now that it is known which plaque components can be identified with ex vivo MR imaging sequences, a translation may be made to in vivo intracranial vessel wall MR imaging to obtain optimal image contrast between the different plaque components. Based on this study, the most promising method for distinguishing these plaque components is T1-weighted imaging.

\section{REFERENCES}

1. Go AS, Mozaffarian D, Roger VL, et al; American Heart Association Statistics Committee and Stroke Statistics Subcommittee, Heart disease and stroke statistics-2014 update: a report from 
the American Heart Association. Circulation 2014;129:e28-e292 CrossRef Medline

2. Lozano R, Naghavi M, Foreman K, et al. Global and regional mortality from 235 causes of death for 20 age groups in 1990 and 2010: a systematic analysis for the Global Burden of Disease Study 2010. Lancet 2012;380:2095-128 CrossRef Medline

3. Arenillas JF. Intracranial atherosclerosis: current concepts. Stroke 2011;42:S20-23 CrossRef Medline

4. Bos D, Portegies ML, van der Lugt A, et al. Intracranial carotid artery atherosclerosis and the risk of stroke in whites: the Rotterdam Study. JAMA Neurol 2014;71:405-11 CrossRef Medline

5. Chimowitz MI, Lynn MJ, Derdeyn CP, et al; SAMMPRIS Trial Investigators. Stenting versus aggressive medical therapy for intracranial arterial stenosis. N Engl J Med 2011;365:993-1003 CrossRef Medline

6. Chimowitz MI, Lynn MJ, Howlett-Smith H, et al. Comparison of warfarin and aspirin for symptomatic intracranial arterial stenosis. N Engl J Med 2005;352:1305-16 CrossRef Medline

7. Gorelick PB, Wong KS, Bae HJ, et al. Large artery intracranial occlusive disease: a large worldwide burden but a relatively neglected frontier. Stroke 2008;39:2396-99 CrossRef Medline

8. Qureshi AI, Caplan LR. Intracranial atherosclerosis. Lancet 2014; 383:984-98 CrossRef Medline

9. Hellings WE, Peeters W, Moll FL, et al. Composition of carotid atherosclerotic plaque is associated with cardiovascular outcome: a prognostic study. Circulation 2010;121:1941-50 CrossRef Medline

10. Saam T, Hatsukami TS, Takaya N, et al. The vulnerable, or high-risk, atherosclerotic plaque: noninvasive MR imaging for characterization and assessment. Radiology 2007;244:64-77 CrossRef Medline

11. Saba L, Anzidei M, Sanfilippo R, et al. Imaging of the carotid artery. Atherosclerosis 2012;220:294-309 CrossRef Medline

12. Takaya N, Yuan C, Chu B, et al. Association between carotid plaque characteristics and subsequent ischemic cerebrovascular events: a prospective assessment with MRI-initial results. Stroke 2006;37: 818-23 CrossRef Medline

13. Kasner SE, Chimowitz MI, Lynn MJ, et al; Warfarin Aspirin Symptomatic Intracranial Disease Trial Investigators. Predictors of ischemic stroke in the territory of a symptomatic intracranial arterial stenosis. Circulation 2006;113:555-63 CrossRef Medline

14. Leng X, Wong KS, Liebeskind DS. Evaluating intracranial atherosclerosis rather than intracranial stenosis. Stroke 2014;45:645-51 CrossRef Medline

15. Dieleman N, van der Kolk AG, Zwanenburg JJ, et al. Imaging intracranial vessel wall pathology with magnetic resonance imaging: current prospects and future directions. Circulation 2014;130:192201 CrossRef Medline

16. van der Kolk AG, Zwanenburg JJ, Denswil NP, et al. Imaging the intracranial atherosclerotic vessel wall using 7T MRI: initial comparison with histopathology. AJNR Am J Neuroradiol 2015;36:694701 CrossRef Medline

17. Majidi S, Sein J, Watanabe M, et al. Intracranial-derived atherosclerosis assessment: an in vitro comparison between virtual histology by intravascular ultrasonography, 7T MRI, and histopathologic findings. AJNR Am J Neuroradiol 2013;34:2259-64 CrossRef Medline

18. Turan TN, Rumboldt $\mathrm{Z}$, Granholm $\mathrm{AC}$, et al. Intracranial atherosclerosis: correlation between in-vivo $3 \mathrm{~T}$ high resolution MRI and pathology. Atherosclerosis 2014;237:460-63 CrossRef Medline

19. Petridou N, Italiaander M, van de Bank BL, et al. Pushing the limits of high-resolution functional MRI using a simple high-density multi-element coil design. NMR Biomed 2013;26:65-73 CrossRef Medline

20. Deoni SC, Rutt BK, Peters TM. Rapid combined T1 and T2 mapping using gradient recalled acquisition in the steady state. Magn Reson Med 2003;49:515-26 CrossRef Medline

21. Yarnykh VL. Actual flip-angle imaging in the pulsed steady state: a method for rapid three-dimensional mapping of the transmitted radiofrequency field. Magn Reson Med 2007;57:192-200 CrossRef Medline

22. Virmani R, Kolodgie FD, Burke AP, et al. Lessons from sudden coronary death: a comprehensive morphological classification scheme for atherosclerotic lesions. Arterioscler Thromb Vasc Biol 2000;20: 1262-75 CrossRef Medline

23. Cai JM, Hatsukami TS, Ferguson MS, et al. Classification of human carotid atherosclerotic lesions with in vivo multicontrast magnetic resonance imaging. Circulation 2002;106:1368-73 CrossRef Medline

24. Cai J, Hatsukami TS, Ferguson MS, et al. In vivo quantitative measurement of intact fibrous cap and lipid-rich necrotic core size in atherosclerotic carotid plaque: comparison of high-resolution, contrast-enhanced magnetic resonance imaging and histology. Circulation 2005;112:3437-44 CrossRef Medline

25. Hofman JM, Branderhorst WJ, ten Eikelder HM, et al. Quantification of atherosclerotic plaque components using in vivo MRI and supervised classifiers. Magn Reson Med 2006;55:790-99 CrossRef Medline

26. Coombs BD, Rapp JH, Ursell PC, et al. Structure of plaque at carotid bifurcation: high-resolution MRI with histological correlation. Stroke 2001;32:2516-21 CrossRef Medline

27. Shinnar M, Fallon JT, Wehrli S, et al. The diagnostic accuracy of ex vivo MRI for human atherosclerotic plaque characterization. Arterioscler Thromb Vasc Biol 1999;19:2756-61 CrossRef Medline

28. Serfaty JM, Chaabane L, Tabib A, et al. Atherosclerotic plaques: classification and characterization with $\mathrm{T} 2$-weighted high-spatial-resolution MR imaging — an in vitro study. Radiology 2001;219:403-10 CrossRef Medline

29. Clarke SE, Hammond RR, Mitchell JR, et al. Quantitative assessment of carotid plaque composition using multicontrast MRI and registered histology. Magn Reson Med 2003;50:1199-208 CrossRef Medline

30. Ritz K, Denswil NP, Stam OC, et al. Cause and mechanisms of intracranial atherosclerosis. Circulation 2014;130:1407-14 CrossRef Medline

31. Chen XY, Wong KS, Lam WW, et al. Middle cerebral artery atherosclerosis: histological comparison between plaques associated with and not associated with infarct in a postmortem study. Cerebrovasc Dis 2008;25:74-80 CrossRef Medline

32. Portanova A, Hakakian N, Mikulis DJ, et al. Intracranial vasa vasorum: insights and implications for imaging. Radiology 2013; 267:667-79 CrossRef Medline

33. Turan TN, Rumboldt Z, Brown TR. High-resolution MRI of basilar atherosclerosis: three-dimensional acquisition and FLAIR sequences. Brain Behav 2013;3:1-3 CrossRef Medline

34. Chung JW, Kim BJ, Choi BS, et al. High-resolution magnetic resonance imaging reveals hidden etiologies of symptomatic vertebral arterial lesions. J Stroke Cerebrovasc Dis 2014;23:293-302 CrossRef Medline

35. Yang WQ, Huang B, Liu XT, et al. Reproducibility of high-resolution MRI for the middle cerebral artery plaque at 3T. Eur J Radiol 2014;83:e49-55 CrossRef Medline

36. Turan TN, Bonilha L, Morgan PS, et al. Intraplaque hemorrhage in symptomatic intracranial atherosclerotic disease. J Neuroimaging 2011;21:e159-61 CrossRef Medline

37. Park JK, Kim SH, Kim BS, et al. Imaging of intracranial plaques with black-blood double inversion recovery MR imaging and CT. J Neuroimaging 2011;21:e64-68 CrossRef Medline

38. $\mathrm{Xu} \mathrm{WH}, \mathrm{Li} \mathrm{ML}, \mathrm{Gao} \mathrm{S}$, et al. Middle cerebral artery intraplaque hemorrhage: prevalence and clinical relevance. Ann Neurol 2012;71: 195-98 CrossRef Medline

39. Yuan C, Mitsumori LM, Beach KW, et al. Carotid atherosclerotic plaque: noninvasive MR characterization and identification of vulnerable lesions. Radiology 2001;221:285-99 CrossRef Medline

40. Lundberg B. Chemical composition and physical state of lipid deposits in atherosclerosis. Atherosclerosis 1985;56:93-110 CrossRef Medline

41. Yuan C, Mitsumori LM, Ferguson MS, et al. In vivo accuracy of 
multispectral magnetic resonance imaging for identifying lipidrich necrotic cores and intraplaque hemorrhage in advanced human carotid plaques. Circulation 2001;104:2051-56 CrossRef Medline

42. Homburg PJ, Plas GJ, Rozie S, et al. Prevalence and calcification of intracranial arterial stenotic lesions as assessed with multidetector computed tomography angiography. Stroke 2011;42:1244-50 CrossRef Medline

43. Sharma S, Boujraf S, Bornstedt A, et al. Quantification of calcifications in endarterectomy samples by means of high-resolution ultra-short echo time imaging. Invest Radiol 2010;45:109-13 CrossRef Medline

44. Thelwall PE, Shepherd TM, Stanisz GJ, et al. Effects of temperature and aldehyde fixation on tissue water diffusion properties, studied in an erythrocyte ghost tissue model. Magn Reson Med 2006;56: 282-89 CrossRef Medline

45. Thickman DI, Kundel HL, Wolf G. Nuclear magnetic resonance characteristics of fresh and fixed tissue: the effect of elapsed time. Radiology 1983;148:183-85 CrossRef Medline

46. Koning W, de Rotte AA, Bluemink JJ, et al. MRI of the carotid artery at 7 Tesla: quantitative comparison with 3 Tesla. J Magn Reson Imaging 2015;41:773-80 CrossRef Medline

47. Pfefferbaum A, Sullivan EV, Adalsteinsson E, et al. Postmortem MR imaging of formalin-fixed human brain. Neuroimage 2004;21: 1585-95 CrossRef Medline 